\title{
Study on Development and Application of Big Data Mobile Terminal on Grid Maintenance
}

\author{
WenYong Yang ${ }^{1, a}$, ZhanFeng Cheng ${ }^{1, b}$, Bo Xia ${ }^{1, c}$, LeLe Pang ${ }^{1, d}$ \\ ${ }^{1}$ Zhangjiakou Power Supply Company, Zhangjiakou, Hebei, 075000 \\ ${ }^{a}$ email, ${ }^{b}$ email, ${ }^{c}$ email, ${ }^{d}$ email,
}

Keywords: Big Data, Mobile Terminal, Grid Maintenance, Application

\begin{abstract}
With the continuous development of power business, customer needs continue to increase, how to improve the efficiency of power supply enterprises, improve service quality and customer satisfaction, has become the power supply enterprises must be addressed. On electrical repairs, how to optimize the customer repair processes, so that the acceptance personnel after receiving a repair call and the faster and more timely information to inform repair personnel, repair personnel after the completion of repair work, more direct information reply to accept people become rather long time to solve repair the key. This paper expounds the main technical, technology roadmap and specific design of system implementation; analysis of the main functions of the system implementation, information on specific design.
\end{abstract}

\section{Introduction}

Power companies supply facilities connected households, over a broad area, with the continuous development of power grid construction, power supply in the area continued to expand, for electrical equipment failure probability is increasing, after the failure of how to do it? If there is no electricity, life will become unthinkable, people's normal life will be greatly affected, and therefore protect the security of supply has become an important safeguard people's lives and work normal social order as well as industrial and agricultural production. As China's power grid construction in full swing, and the level of the whole society electricity supply and improve customer service needs, to meet the power scientific, economic, efficient operation, we must improve the automation level of the grid, while the power supply enterprise service levels We must also keep. Therefore, to maintain and ensure the safety of the power supply network to ensure that the customer's normal use of electricity is very important. If only a high level of automation of the grid, and the lack of high-quality and efficient after-sales service means, the power supply enterprise is flawed. So if a power failure repair in a timely manner is an important indicator to judge the level of power supply enterprise service.

Power fault repair system is the use of mobile devices, combined with failure to repair fast and convenient fault repair processing systems business itself is characterized by the development, through information on the fault repair work, standardized operation, establish a convenient electricity fault repair work, reliable information exchange platform for fault repair personnel complete failure to repair information collection, repair the daily operation of data processing, the establishment of a power failure repair information database, completely changed in the past before troubleshooting, back office staff to describe the same fault is not uniform, processing report such problems as varied, so that the power fault repair work information, efficient. And reduce labor intensity of staff, improve the quality of work, based on the needs of fault repair system of a mobile terminal is urgent.

\section{Requirement of System Analysis}

Establishment of mobile data platform, 95598 Repair System data mobile, mobile terminal application in the upcoming repair system, to streamline operations, customer service staff to facilitate communication and field repair personnel, thereby making repair personnel faster and more accurate grasp the point of failure information in a timely manner to achieve the scene to 
complete the repair work. After repair the situation, it is possible to accurately feedback through the mobile terminal to the customer service staff, reduce information errors, improve customer satisfaction, the use of the system reconstruct fault repair process. Require the use of a mobile terminal to establish a mobile data exchange platform, to achieve mobile switching 95598 Repair System data, reducing staff, reducing the flow part of the staff into the repair work, contact customer service personnel also from customers waiter - repair scheduling - repair personnel, into direct contact with the customer service repair personnel, improve work efficiency.

The system is mainly for Jinhua Electric Power Bureau Customer Service Center 95598, Jinhua Electric Power Bureau under the county branch of the line repair personnel Jinhua Electric Power Bureau and online marketing managers, technical requirements are as follows: 1 , should follow the standards and specifications: Zhejiang Electric power company system should follow the promulgation of "GPRS/CDMA common gateway Interface standard" requirement; the interface between the provincial 95598 customer service system and application server, to comply with the standard HTTP protocol; follow the provincial power system privileges 95598 user rights, another portion Let users. 2, the characteristics of the system requirements: timeliness: timely access to first-line repair personnel repair work orders for specific information and timely feedback. Mobility: first-line repair personnel to exchange information in a mobile data state. Flexibility: supporting the mainstream market Mobile and Android operating system currently.

Jinhua Electric Power Bureau Customer Service Center aims to establish a technical support system for front-line service personnel power system to facilitate the working conditions in the mobile state, reduce the amount of labor information processing, improve service levels and efficiency, and strive to achieve electronic work, fast technology. Maintenance requirements the system is simple, fast and clear process. Ticket inquiries have specific needs, work order processing, site upload photos, GPS positioning, interface requirements.

\section{The Overall Design Concept of the Grid Maintenance System}

After using the mobile terminal to establish a mobile data exchange platform, and the mobile switching 95598 Repair System data. Repair scheduling link is omitted, which can reduce staff, but also reduce the flow of links, can be put into the repair work, contact customer service personnel from the original customer service - scheduling repairs - repair personnel, became a customer direct contact with the waiter repair personnel, greatly improving the work efficiency.

Power system through the mobile terminal mobile applications, mainly following requirements threefold: to get anywhere, collect required information; the need to extend and expand the power system information; the need to enhance the power line service personnel service capabilities and rapid response capability. Solve the problems of the current model: The traditional way is not timely send a single, time-consuming and tedious work; send a single lack of effective monitoring, affect the quality of customer service and reputation; after repair of feedback slow and inaccurate information; customer service to send a single volume, lack of control headquarters, headquarters difficult to verify the accuracy of the work order information; manual processing, information is not easy to make a comprehensive feedback.

When the user use the mobile terminal, will encounter a variety of problems for all sorts of problems and to provide an effective method of preventing the loss of important information. Mainly in the following security policy: Parity: ESN/IMSI codes, mobile terminal number binding, a mobile terminal card, together with the mobile terminal landing mobile 95598 system. To prevent exhaustive self-destruction: mobile office system only allows the user to login attempts password three times, if not right, it will automatically suspend a user's connection. It makes it impossible to complete the user's password brute-force attack. If forced to enter the password again, the system will automatically self-destruct, delete all SMS client terminal information and the mobile terminal, contacts, and other related confidential information, the first time to ensure the confidentiality of information. Time expired: when the mobile terminal and the server communications terminal is not within a set period of time, that is, when there is no mobile terminal users to operate the mobile terminal within the set time, the system default mobile terminal may have to exit the system, the 
server automatically closes the connection mobile terminal terminal can not carry out any operation.

The mobile terminal architecture consists of a custom server (EMA) and the mobile terminal client and we need to take advantage of China Telecom CDMA 3G data network through the application gateway provincial CDMA communication network.

The system software platform: WEB application platform of this system is TOMCAT.TOMCAT is a small, lightweight application server, is widely used in many occasions are not small and medium systems and concurrent access, the development and debugging JSP procedures preferred. Database system is an important part of this system, database platform must have the following characteristics: good client/server architecture, heterogeneous data source access capability, high reliability and security, good WEB SERVER function, support a large number of online users, providing distributed data and control, support third-party GUI development tools including CASE, database maintenance tools, Client-side development tools, query development tools. Taken together, this system uses the Postgre SQL database system. System design using xMAP middleware agent accessible mode completes the relevant functions. XMAP is intermediate plug products from xMAP-C (client), xMAP-S (server), xCOM (communication capability) three modules, the overall framework for the use of C/S architecture, the establishment of loosely coupled connection with third-party system, and the whole adaptation conversion process. xMAP-C core modules include application management, rendering engine, smart upgrade agents, agents and other local capacities, including application management is the highlight of xMAP-C function, multi-application support, and xMAP-C and application separation. Furthermore, xMAP-C also introduces object scripts for logic implementation of the settlement IT systems control script adds support local database to assist in improving the efficiency of data reading and writing, through close cooperation with Local database and core processing module allows the client data and operations in the integration, to play a greater role in the smart client. xMAP-S on the basis of the system platform, required to achieve SI Toolkits tool design package, Basicability basic capabilities, Multi-protocol multi-protocol adapter, Business Statistics Business Statistics of the four major functional blocks. Foreign applications have access interface, terminal access interface, the communication interface of the three core interface. xCOM: using xMAP communication module, with SMS, MMS, wappush peer communications capabilities. 3, the system is running: The uixml second extension agreed set of tags and attributes, the business content server PC interface through data analysis and logic analysis, 2G/3G network transmission to the client display, complete PC-to-mobile information terminal of. 4, based on the successful experience in the information field of construction, the system design based on EMA (Enterprise Mobility Agent) Mobile reporting solutions, has deployed mobile office server room within the enterprise, combined with existing applications, these applications extending from the PC terminal to the mobile terminal solutions. Ensure mobile terminal system configuration parameters intact, able to exchange data via telecommunications CDMA network and provincial corporate gateway.

Long established TCP connection between the server and gateway EMA as "client", log on to the gateway to receive data and occurrence. EMA is binding relationship configuration server's IP address and the logical address of the gateway. Mobile client is when you start the program, and the establishment of long-gateway connections, when you exit the program, disconnect and gateway connection. The original packet data communication between the phone and the server EMA, with FAAL protocol package again. On the mobile client and server communication module EMA increase unpacking and pack of logic.

\section{Conclusion}

Big data is an emerging field of practical significance and challenging, has great potential value, the continuous development of big data technology and its application in various fields, will the future development of China's electric power industry, especially the smart grid It is an extremely important and far-reaching impact on construction and development. In the field of power system substation maintenance, thinking big data facilitate the integrated use of substation maintenance strategy, substation maintenance will help improve the level and efficiency. 


\section{References}

[1] GaLLupe B. Knowledge Management Systems: "Surveying the Llsndscape" [J] International Journal of Management Re2views 2002.1 (3): 61277

[2] Bluetooth SIG Specification of the Bluetooth System, Version 1.Ob .WWW.bluetooth.com, Dec1, 1999

[3] Currie, W, Galliers, B. Rethinking Management Information Systems [J] .New York:. OxfordUniversity Press Inc, 1999, Vol.1: 56-73

[4] Broderic, R, Boudrean, J. Human Resource Management, Information Technology, and the Competitive Edge [J] .Academy of Management Executive, 1992, Vol.6 (No2): 7-17

[5] Guichard, Jim/Pepelnjak, Ivan/Apcar, Jeff .Mpls and Vpn Architectures [M]. Macmillan T echnical Pub 2005, 7

[6] Alwayn Vivek. Advanced MPLS Design and Implementation[M]. Indianapolis, IN: Cisco Press, 2002. 